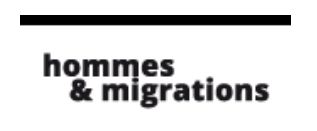

Hommes \& migrations

Revue française de référence sur les dynamiques

migratoires

$1326 \mid 2019$

Londres et ses migrations

\title{
Collectionneur de mots, les maux du collectionneur
}

\section{Hubert Cavaniol}

\section{(QpenEdition \\ Journals}

\section{Édition électronique}

URL : https://journals.openedition.org/hommesmigrations/9793

DOI : 10.4000/hommesmigrations.9793

ISSN : 2262-3353

\section{Éditeur}

Musée national de l'histoire de l'immigration

\section{Édition imprimée}

Date de publication : 1 juillet 2019

Pagination : 88-92

ISBN : 978-2-919040-46-9

ISSN : $1142-852 X$

\section{Référence électronique}

Hubert Cavaniol, «Collectionneur de mots, les maux du collectionneur », Hommes \& migrations [En ligne], 1326 | 2019, mis en ligne le 01 juillet 2019, consulté le 15 janvier 2022. URL : http:// journals.openedition.org/hommesmigrations/9793 ; DOI : https://doi.org/10.4000/ hommesmigrations.9793 
PORTFOLIO

\section{Collectionneur de mots, les maux du collectionneur

Hubert Cavaniol, responsable du prêt des œuvres et de la coordination des expositions du Petit Palais (Paris).

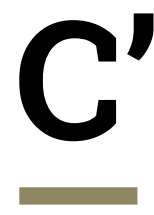

est aux Puces de Clignancourt que tout a commencé, il y a vingt ans. Par hasard, j'y achetai une affiche de cinéma évoquant la frontière. Quelque temps après, au même endroit, j'en trouvai une deuxième, celle du film Contrebande au Caire. Des années plus tard, ce seront bel et bien des centaines d'autres d'affiches qui me traceront le chemin vers le Musée national de l'histoire de l'immigration.

Par goût, je collectionne les mots. Par curiosité, je me suis intéressé à ceux évoquant la frontière dans le cinéma. Tous les mots dont les titres faisaient rêver : 《frontière », bien évidemment, mais aussi « douane », 《contrebande », 《 douanier », 《 contrebandier 》, 《 visa 》, " passeport 》, 《trafiquants 》, 《trafics 》... D'improbables films sortaient de leur oubli: Contrebande à Shanghai, Passeport pour la honte, Les contrebandières, La fiancée du douanier, Chiens de contrebandier, Passeurs d'or, Porte d'Orient, Filles de fraudeurs... Une belle affiche, c'était d'abord un titre, une typographie puissante. Les esquisses gouachées des grands affichistes de l'époque, Jean Maschii (1926-2003), Jean Ferracci (1927-1982), Constantin Belinsky (1904-1999), devenaient d'immenses lithographies explosant de couleurs, emplies de postes frontières, de lumières lugubres, de femmes fatales au bras de contrebandiers fiévreux, de ports brumeux, de douaniers soupçonneux... Il ne s'agissait pas de s'intéresser particulièrement à la qualité cinématographique d'un film, mais plutôt de goûter à la pure émotion dégagée par l'affiche, à son épaisseur visuelle, à cette autre dimension qui pouvait la transformer en un objet.

La recherche par mots avait cependant ses limites : pourquoi le film Tip on a Dead Jockey $^{1}$ était-il sorti en France sous le titre plus accrocheur de Contrebande au Caire? Ce que je cherchais n'était que l'écume du genre, il fallait se plonger dans le sujet. D'abord commencer par dépouiller les 15000 notices de films du Guide des films de Jean Tulard qui recensait «tout ce qui a compté dans la production cinématographique d'un point de vue occidental »; puis, compléter ces premières recherches par la lecture de tous les Index de la cinématographie française édités 
chaque année depuis 1947; croiser les informations recueillies avec celles des Années du cinéma, du Guide des 10000 films ou de l'Histoire du cinéma français en 6 volumes... Mais par-dessus tout, j'aimais filer à la Bibliothèque du film - installée alors rue Saint-Antoine -, pour y passer de délicieuses et studieuses après-midi à rédiger des fiches, établir des listes, traiter des milliers d'informations pour comprendre et évaluer l'étendue du sujet. La matière était riche, la contrebande avait eu son âge d'or, elle était presque devenue un genre cinématographique dans la période 1910-1960; quel bonheur de découvrir Ginette Leclerc ${ }^{2}$, les films d'Émile-Georges De Meyst ${ }^{3}$ - l'un des maîtres du genre -, et une incroyable filmographie peuplée d'acteurs, d'actrices et de metteurs en scène, pour la plupart aujourd'hui oubliés. En cinq ans, j'avais réuni plusieurs centaines d'affiches de cinéma sur le thème de la contrebande, mais aussi plus largement sur celui de la frontière, presque tout ce qu'il était possible de trouver en France sur ce sujet compte tenu des inévitables contraintes matérielles auxquelles se trouvait confronté le collectionneur compulsif. Bien évidemment, tout cela ne servait à rien, n'avait aucun sens, mais comme l'avait dit le grand collectionneur anglais Sir Edwin Manton (1909-2005), «It's better than spending your money on bottles of Scotch. $\left.{ }^{4}\right\rangle$

\section{De Contrebande au Caire à Bicots-Nègres, nos voisins}

Et puis un jour, arrive ce qui paraît impensable, la quête essentielle devient négligeable, «le tour du sujet » semble avoir été fait, et l'on se retrouve seul face à la vacuité d'un amoncellement d'objets devenus inutiles. Pire, je découvrais avec consternation que je n'avais rien vu des hommes et des femmes qui passaient les frontières, dans des conditions parfois effroyables. J'ai voulu alors rattraper le temps perdu en allant aussi loin que je le pouvais dans l'étude des migrations au cinéma ; il était important de collecter et de transmettre cette mémoire de papier. Avec le cinéma, j'avais découvert un art. Avec les migrations, j'ouvrais les yeux sur les sciences sociales. En croisant les deux, un abîme d'ignorance s'ouvrait, le mien. Et pourtant, c'est cette ignorance qui m'a porté. Il fallait encore lire l'essentiel de ce qui avait été publié, mais là, c'était impossible, il y avait tellement de livres, d'analyses, de thèses, d'articles... C'était un autre monde, celui des revues spécialisées, des festivals, des sites Internet, des associations, des musées, de l'engagement de l'État dans le secteur cinématographique. Comment raconter la jubilation de l'amateur quand, sur un rayonnage de la librairie de L'Harmattan', il trouve le livre Cinémas de l'émigration ${ }^{6}$ ? Sur la quatrième de couverture, en quelques lignes, tout était dit pour m'éclairer : "Si l'on excepte Toni", consacré en 1934 par Jean Renoir aux travailleurs italiens en France, le cinéma a tardé à rendre compte de ce phénomène. Depuis dix ans, pourtant, plus d'une centaine de films de tous métrages et de tous formats, ont été tournés, d'abord par des militants d'avant-garde puis par des cinéastes établis pour dénoncer le statut de métèques où sont confinés les

2. Chemins sans loi, France, 1947, de Guillaume Radot; Passeurs d'or, France, 1948, de E. G. De Meyst; Les aventuriers de l'air, France, 1950, de René Jayet; La maison dans la dune, France, 1952, de René Jayet.

3. Filles de fraudeurs, France, 1962; Passeurs d'or, France, 1948.

4. Citation extraite de la biographie de Sir Edwin Manton lue sur les cimaises du Sterling \& Francine Clark Art Institute de Williamstown, Massachusetts, États-Unis.

5. La seule découverte de cette librairie avait déjà été en soi un choc.

6. Guy Hennebelle, Cinémas de l'émigration, Paris, Filméditions, 1979.

7. Toni, 1935, France, de Jean Renoir. 
Bicots-nègres, nos voisins ${ }^{8}$ par l'inconscience de trop de Dupont Lajoie 9 . » Vingt ans après, Guy Hennebelle avait toujours raison: à part le Toni de l'avant-guerre, la filmographie française était maigre. Par où commencer? Guy Hennebelle avait été précis, pourquoi s'embarrasser? Il avait dit 100 films. Cela faisait 100 affiches à trouver. L'aventure pouvait démarrer. Elle s'est arrêtée à 2000 affiches en 2018, le sujet a été plus vaste que prévu.

Sur tous les continents, dans tous les pays, sur les mers et sur les océans, les souffrances et les espérances des migrants avaient été portées sur les écrans, et cela continuait. "De salles en salle, la silhouette du réfugié hante le festival (de Cannes). Ils apparaissent chaque jour, au détour d'un plan ou d'un plein écran, depuis le début de ce festival ${ }^{10}$. » Il y avait les migrations, mais aussi le racisme, l'esclavage, la question de l'altérité, le colonialisme, la guerre d'Algérie, les banlieues, l'intégration, le «voyage »... Dans un premier temps, je me tournai vers la France, puis vers le Maghreb, en ciblant tout particulièrement les films des trois décennies 1960-1990; un ensemble de 100 affiches de cette période a formé l'essentiel de cette première donation au Musée national de l'histoire de l'immigration. Ensuite, l'Italie, ses migrations du Sud vers le Nord, puis vers l'Europe du Nord, et les grands classiques du cinéma transalpin Il camino delle speranza ${ }^{11}$, Pane e cioccolata ${ }^{12}$, Padre Padrone ${ }^{13}$; puis l'Allemagne et la question de l'immigration turque avec l'introuvable (...mais trouvée) $40 \mathrm{~m}^{2}$ Deutschland ${ }^{14}$, et l'incontournable Angst essen Seele auf ${ }^{15}$. Et tout le reste de l'Europe. J'étais submergé.

Plus tard, je découvrirai les États-Unis et la question de l'immigration omniprésente sur les écrans américains depuis les débuts du cinéma. C'était considérable. Mais aussi désespérant. Comment espérer acquérir les affiches originales des films The Immigrant ${ }^{16}$ ou Birth of a Nation ${ }^{17}$ ? Comment aborder la question des Natives, celle du mythe fondateur de la «frontier», de la Conquête de l'Ouest et du massacre des Indiens, de l'arrivée des Européens depuis le XVI e siècle? Par la suite, j'étudiais la ségrégation, la question raciale, la présence de la figure du noir, la color line, la Blaxploitation, Cassius Clay. Les noirs américains m'occupèrent longtemps.

\section{Du Passage Jouffroy à Nakano Broadway}

Avant de poursuivre, il faut parler de l'outil indispensable dont ne se sépare jamais le collectionneur de vieux papiers : la liste. Que ce soit une simple feuille de papier froissé sortie d'un portefeuille usé, un calepin de cuir rempli d'une fine écriture, ou bien une liasse de format A4 soigneusement agrafée et pliée, emplie de centaines de lignes d'un tableau Excel, un collectionneur avisé se promène toujours avec « sa » liste. En quelque sorte une passion que l'on emporte avec soi et que l'on porte sur

\footnotetext{
8. Les Bicots-Nègres, vos voisins, 1974, Mauritanie/France, de Med Hondo.

9. Dupont-Lajoie, 1975, France, d'Yves Boisset.

10. Le Monde, 2003

11. Il camino della speranza (Le chemin de l'espérance), Italie, 1950, de Pietro Germi.

12. Pane e cioccolata (Pain et chocolat), Italie, 1974, de Franco Brusati.

13. Padre Padrone, 1977, Italie, de Paolo Taviani et Vittorio Taviani.

14. $40 \mathrm{~m}^{2}$ Deutschland (40 $\mathrm{m} 2$ d'Allemagne), Allemagne, 1986, de Tevfik Baser.

15. Angst essen Seele auf (Tous les autres s'appellent Ali), Allemagne (RFA), 1973, de Rainer Werner Fassbinder.

16. The immigrant (L'immigrant), USA, 1917, de Charlie Chaplin.

17. The Birth of a Nation (Naissance d'une nation), de D. W. Griffith.

Un ensemble de deux affiches US 1sh et US 1sh «Klansman » s'est vendu 67300 dollars le 14 décembre 2012 sur le site de vente aux enchères emovie.com.
} 
son cœur. Mais cette liste, autrefois expression d'un travail de recherches parfois considérable, n'est plus que l'aboutissement de croisements de bases de données. Quand il fallait un temps infini pour espérer appréhender un sujet, l'essentiel peut être dit aujourd'hui en deux temps, trois clics: sur la base IMDB.com, le mot «immigration》 fait ressortir 1291 titres; le mot «immigrant》, 1616. C'est beaucoup. L'excellent site cinefiches.com répertorie quant à lui près de 400 films, classés par thèmes/problèmes de société/pays. À titre de comparaison, le mot « contrebande» fait sortir 2368 titres sur IMDB.com. Ensuite, c'était le plaisir de « faire du papier », l'obligation de se retrousser les manches face aux piles d'affiches, les relations étroites nouées avec les marchands, la lecture des catalogues de ventes aux enchères, l'attente des rendez-vous annuels qui réunissaient tous les amateurs et les professionnels à l'occasion des grands-messes qu'étaient alors Champerret et son salon du Vieux papier, Argenteuil et ses Cinglés du cinéma, Cormeilles-enParisis et sa Bourse Photo-Ciné, sans négliger toutes les brocantes spécialisées, les bourses de collectionneurs dont l'objectif est de « donner l'occasion d'acheter, de vendre et d'échanger des affiches, photos, appareils ciné, projecteurs, autographes (...) ${ }^{18}$ ॥. Il y a encore quelques années, plusieurs galeries spécialisées sur le cinéma tenaient le haut du pavé à Paris. Il y avait les deux formidables « barbus » du Passage Verdeau près de Drouot qui m'ont tout appris sur le cinéma africain. C'est chez eux que j'achetais mes premières et rares affiches des films de Med Hondo, Sydney Sokhona ou bien Djibril Diop-Mambety «...avec vous, elles seront en de bonnes mains 》. Il y avait aussi les grands marchands, vedettes de leur catégorie, Stanislas Choko de la galerie Intemporel aux Halles et Jean-Louis Capitaine de la galerie Ciné-Images, rue de Babylone ${ }^{19}$, qui, lorsqu'il m'appelait pour me dire: « Hubert, j'ai un truc pour toi», me faisait arriver dans l'heure. Tous ceux dont le nom s'est envolé, mais dont je me souviens parfaitement; ils exerçaient rue Broca, rue Saint-Martin, aux Puces de Clignancourt, rue des Blancs-Manteaux... De ces galeries dédiées au seul cinéma, deux subsistent à Paris. D'autres marchands avec qui le dialogue est toujours un plaisir, Alain, Alexandre - qui me trouva, après dix ans d'une vaine quête, la magnifique affiche du film Moi, un Noir ${ }^{20}$ de Jean Rouch -, Benoît, tous cinéphiles passionnés et d'une culture stupéfiante. L'essentiel se vend cependant aujourd'hui sur Internet.

Chercher ailleurs? C'était évident. En France, il existait de bons marchands. Mais aussi à Londres, avec la très chic Reel Poster Gallery où la présentation des affiches laissait accroire que l'on pouvait être chez un joaillier de la Place Vendôme, Bruxelles, Amsterdam, Barcelone et Madrid, Rome (et la miraculeuse découverte d'un immense entrepôt près de la Gare Termini), New York et ses mythiques marchands Philip William, Posteritati, mais surtout Chisholm Larsson et son slogan "Chissholm Larsson has more than 60000 posters ». Chicago, Los Angeles, Toronto... toutes les villes où mon métier me faisait partir. Tokyo et sa mine de livres du quartier de Jimbocho où plusieurs centaines de libraires se serrent dans quelques rues. Dans ce quartier, qui n'a pas d'égal au monde, on trouve encore trois librairies spécialisées dans le cinéma. L'anglais y est une langue rare; le français, une langue morte. Y trouver une affiche de cinéma sur l'immigration relève de l'exploit, se prépare bien avant de pousser la porte. À Tokyo toujours, des marchands découverts après d'éreintantes marches, et qui l'année d'après n'existent plus pour

18. Flyer publicitaire de la 8 e bourse de collectionneurs cinéma à Anvers, Belgique, 12 mars 2006

19. Jean-Louis Capitaine et Stanislas Choko sont décédés.

20. Moi, un Noir, 1958, France. 
le commun des promeneurs, comme mystérieusement gommés de la mémoire du quartier. Pour les amateurs aventureux, il ne faudra jamais oublier de passer à Nakano Broadway, insondable centre commercial planté en lointaine banlieue et dont la découverte est à elle seule une récompense. Vous y trouverez, perdues dans les étages, deux minuscules boutiques bourrées d'affiches de cinéma. Flâner dans Nakano Broadway m'a certainement plus appris sur la vie japonaise que les incontournables visites du temple de Sensō-ji.

\section{Jusqu'à Tarkovsky}

Les affiches arrivaient en nombre. Sans même creuser, j'ai toujours trouvé de nouveaux murs, de nouvelles frontières: en France, la Ligne Maginot, la Ligne de démarcation, le Mur de l'Atlantique, aujourd'hui Calais ; ailleurs, la Grande Muraille de Chine, le Rio Grande, le $38^{\mathrm{e}}$ parallèle, le Mur de Berlin, le Rideau de Fer, la Ligne verte, les enclaves espagnoles de Ceuta et Melilla, le mur de Gaza... « Toujours plus de murs dans un monde sans frontières ${ }^{21}$. » Toutes ces murailles visibles et invisibles avaient été portées à l'écran comme si la barque n'était pas assez chargée, surtout ne pas oublier Kennedy et sa Nouvelle Frontière, Jules Verne et ses romans de tous les mondes, Andreï Tarkovsky et son chef-d'œuvre Stalker ${ }^{22}$, sa Zone et sa chambre magique, ses sublimes affiches effrayantes... Je sombrais. Pourquoi s'arrêter en 2019 ? Pour me libérer. Pour transmettre. Il m'a aussi semblé plus sage de laisser ce sujet aux spécialistes du septième art, les migrations (les "phénomènes » migratoires), comme le western le fut à son époque, étant devenues un nouveau genre cinématographique. "Nous allons voir des films qui assument leur statut de fiction, mais parce qu'ils sont fidèles à ce qu'ils représentent, ils sont plus puissants, plus proches de la réalitée ${ }^{23}$.»

Mais la première affiche, celle des Puces de Clignancourt? Ce jour-là, j'avais pensé faire plaisir à mon épouse Béatrice en lui offrant l'affiche du film Vous n'avez rien à déclarer ? $^{24}$. Elle avait été tellement désolée de me dire qu'il n'était pas possible de l'accrocher dans son magnifique bureau en rotonde situé à l'angle des rues du Bac et de Montalembert. Elle travaillait à la Direction générale des douanes françaises ; l'affiche représentait l'impayable Darry Cowl déguisé en douanier tenant au-dessus de sa tête une valise grande ouverte. C'est ensuite que cette histoire a commencé.

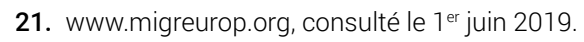

22. Stalker, Union Soviétique, 1979, d'Andrei Tarkovsky.

23. Le Monde, 15 mai 2019, p. 21, entretien avec Alejandro Gonzalez Inarritu.

24. Vous n'avez rien à déclarer?, 1959, France, de Clément Duhour. 